\section{How older persons structure information in the decision to seek medical care}

\author{
Peter J. Veazie \\ Department of Public Health Sciences, \\ University of Rochester, NY, USA
}

\section{Abstract}

Typical models of the decision to seek care consider information as a single conceptual object. This paper presents an alternative that allows multiple objects. For older persons seeking care, results support this alternative. Older decision-makers that segregate information into multiple conceptual objects assessed separately are characterized by socio-demographic (younger age, racial category, non-Hispanic, higher education, higher income, and not married), health status (better general health for men and worse general health for women, fewer known illnesses), and neuropsychological (less memory loss for men, trouble concentrating and trouble making decisions for men) factors. Results of this study support the conclusion that older persons are more likely to integrate information, and individuals with identifiable characteristics are more likely to do so than others. The theory tested in this study implies a potential explanation for misutilization of care (either over or under-utilization).

\section{Introduction}

Models of decision making often assume the integration of information by which individuals seek care when the weighted sum of evaluated informational elements, or some function thereof, exceeds some threshold.1,2 These are compensatory models in which trade-offs can occur between attributes. Other models are non-compensatory in which such trade-offs are not made but rather judgments and decisions are better described by heuristics or rules.3-5 And still other models endeavor to integrate both approaches. ${ }^{6}$ In these models the objects of assessment are those entities about which judgments and decisions are being make. Is this person ill? Which treatment should be used? Which physician has more expertise? In this paper the objects are the conceptual complexes that represent information about the judgment or decision problem. For example, a patient exhibiting symptoms related to different physiological systems is seen by an emergency room physician who may decide whether immediate action is required for either of 2 indicated problems (assessed separately): is immediate care required for nerve damage? Is immediate care required for skeletal damage? Or the decision may be based on the overall threat implied by the injuries considered together. The principal proposition of this paper is that a decision is based on separate assessments of these distinct cognitive objects: each object comprising a network of related concepts. The assessment of each object can be accomplished by any information processing model whether compensatory, non-compensatory, or a combination of both. Knowledge of the kind relevant here comprise elementary concepts (e.g., Medication and Headache) and relational concepts (e.g., Medication X causes headaches). ${ }^{7}$ Disjoint groups of consciously-related concepts are termed conceptual complexes; 8 they are the objects of deliberation, the content of decisions. Consider the example presented in Figure 1. Panel 1 depicts a complex with eleven elementary concepts labeled $A$ through $K$ : Eye strain (A), Stroke (B), Reading (C), Headache (D), Medication being taken (E), Chest Pain (F), Heart attack (G), Heart disease (H), Arm pain (I), Aging $(\mathrm{J})$, and Indigestion (K). A decision based on this complex would account for all concepts. If, however, the relational concept connecting $D$ and $E$ is deleted, then the two distinct conceptual complexes shown in Panel 2 are generated: $\{A, B, C, D\}$ and $\{E, F$, $G, H, I, J, K\}$. In this case, because the decision maker does not consider a relationship between the medications and the headache, a decision is made if either remaining complexes is sufficient. If the elementary concept $G$ is also deleted (i.e. the decision maker does not consider heart attack in their conceptualization of the problem), then the three complexes shown in Panel 3 result. If the complex defined by the set $\{E, F, G, H\}$ is deleted from the general complex shown in Panel 1 (i.e. the decision maker does not consider heart disease, heart attack, medication, or chest pain), then the two complexes shown in Panel 4 result.

The present study has two research questions: i) Are older persons more likely to structure information in the decision to seek medical care as a 1-complex or a multi-complex model? ii) Which characteristics differentiate the relative likelihood of the 1-complex model from the multi-complex model? The second question is elaborated in terms of the following hypotheses regarding the following characteristics.

\section{Age}

Aging corresponds to decreased inhibition of irrelevant information. ${ }^{9,10}$ In the terms of the presented theory, this implies conceptual complexes can be filled out with what should otherwise be irrelevant concepts. This sug-
Correspondence: Peter J. Veazie, Department of Public Health Sciences, University of Rochester Medical Center, Department of Public Health Sciences, 265 Crittenden Blvd., CU 420644, Rochester, NY 14642-0644, USA.

Tel.: +1.585.273.5464 - Fax: + 1.585.461.4532

E-mail: peter_veazie@urmc.rochester.edu

Key words: aging research, help seeking, information processing, decision-making, medicare current beneficiary survey.

Acknowledgements: this manuscript is based on the author's PhD dissertation work at the University of Minnesota that was supervised by his committee: Mike Finch PhD, Robert Kane MD, Sandy Potthoff PhD, Paul Johnson PhD, and Sudipto Banerjee PhD.

Conflict of interests: the author declares no potential conflict of interests.

Received for publication: 28 March 2014.

Accepted for publication: 26 April 2014.

This work is licensed under a Creative Commons Attribution NonCommercial 3.0 License (CC BYNC 3.0).

(C) Copyright P.J. Veazie, 2014

Licensee PAGEPress, Italy

Health Psychology Research 2014; 2:1535

doi:10.4081/hpr.2014.1535

gests that otherwise separate conceptual complexes can be bridged by irrelevant concepts. Aging also corresponds to greater inaccuracy in constructive memory, ${ }^{11}$ decreased episodic memory in terms of both item and associative recall, 12 and decreased ability to maintain concepts in working memory. ${ }^{13}$ Hence, older persons are less able to appropriately recall and maintain elementary and relational concepts, suggesting conceptual complexes are less likely to be merged by appropriate relational concepts. Hence, the hypothesis is that age among the elderly differentiates models with fewer complexes from those with more conceptual complexes.

\section{Sex}

Men and women differ in performance on various cognitive tasks. ${ }^{14}$ At the neurological level the brain functions differently, particularly the prefrontal cortex; this manifests as women recalling and incorporating more concepts in cognitive operations. ${ }^{15}$ In terms of the present theory, women may generate more elementary and relational concepts than men that can connect otherwise disparate complexes, implying the hypothesis that women have a greater likelihood of generating fewer conceptual complexes rather than more complexes. 


\section{Culture}

Performance on decision-related tasks vary across cultures, ${ }^{16}$ particularly in the areas of risk perception, ${ }^{17}$ self-confidence in judgment, ${ }^{18}$ and decision processes. ${ }^{19}$ At a cognitively deeper level, culture differentiates knowledge structures such as schemas and implicit theories. ${ }^{20}$ Knowledge structures influence what information is encoded in memory, how information is interpreted and integrated in terms of its relationships to extant knowledge, and how mental representations are retrieved or reconstructed. ${ }^{21}$ The links between culture, schemas, and mental representations implies that culture influences mental representations and the organization of conceptual complexes. The consequent hypothesis is that culture differentiates models of fewer versus more conceptual complexes.

\section{Education level}

Education facilitates acquisition of concepts and the structuring of cognitive processes: 22 both are relevant to construction and organization of conceptual complexes. Evidence indicates education relates to differential brain structure and information processing. ${ }^{23,24}$ The presumption is different educational backgrounds correspond to different mental representations of decision environments and differently structured conceptual complexes. The corresponding hypothesis is education differentiates models with fewer versus more conceptual complexes.

\section{Financial status}

Financial status represents a component of socio-economic status that captures the effect of socialization on the structuring of information. People with greater means engage different or expanded social groups: the first being the case where income defines the social group within which an individual directly socializes; the second being the case where income provides the ability to access information from particular groups. The hypothesis is that financial status differentiates models of fewer versus more conceptual complexes.

\section{Number of illnesses}

Those with more known illnesses are presumed to have had greater exposure to illness information and are likely to more accurately generate relational concepts that tie signs and symptom together. The hypothesis is that a greater number of illnesses corresponds to a higher likelihood of forming fewer conceptual complexes.

\section{General health and physical functioning}

It is presumed that decreased health and physical functioning provide the impetus for reflection on health state and consequently refining the schema that underlie judgments about current information and the decision to seek care. It is hypothesized that perceived health status and level of physical functioning differentiates models of fewer versus more conceptual complexes.

\section{Cognitive functioning}

Diminished capability in memory, concentration, and decision-making is presumed to signify fundamental changes in cognitive functioning. Though it does not necessarily follow that the structure of conceptual complexes must be affected, the proposition is plausible. It is hypothesized cognitive functioning differentiates models of fewer versus more conceptual complexes.

\section{Social support}

This study focuses on two sources of social support: the presence of a spouse, ${ }^{25}$ and number of children. ${ }^{26,27} \mathrm{~A}$ spouse and children are likely to have intimate knowledge of, and influence in, a person's deliberations regarding health care. The hypothesis is that the presence or absence of a spouse and number of children will differentiate models of fewer versus more conceptual complexes.

\section{Materials and Methods}

\section{Data}

Two consecutive years, 1996 and 1997, of the Access to Care data of the Medicare Current Beneficiary Survey (MCBS) are used for this analysis. The MCBS is sponsored by the Centers for Medicare and Medicaid Services; it is a continuous, multipurpose survey of a nationally representative sample of Medicare beneficiaries. The MCBS provides information on health status, health care use and expenditures, health insurance coverage, and socioeconomic and demographic characteristics of beneficiaries. The first year of data used for this analysis (1996) provides the explanatory variables, and the second year (1997) provides both the decision to seek care variables and the exclusion criteria variables.

Respondents are excluded from analysis if their reason for seeking medical care during the survey period was that they were instructed to seek care by a health care provider (e.g. their visit was a follow up appointment or referral); respondents for whom a reason for seeking medical care was not provided were also excluded. Respondents are excluded if they were institutionalized (e.g., nursing home) within the preceding year. Proxy responses were not included. Analysis was performed on 2020 beneficiaries.

\section{Measures}

\section{Decision to seek care}

The decision to seek medical care was measured as an indicator that the patient visited the doctor's office on the patient's initiative and not as a provider requested referral or follow-up visit.

\section{Predictors of the decision to seek care}

The variables used to predict the decision to seek care (denoted by $x$ in equations 2 and 3 below) include age, sex, race, ethnicity, education, income, marital status, difficulty writing, memory loss, difficulty in making decisions, and number of known illnesses. These variables were selected from a larger set of potential predictors using a stepwise process to retain variables with P-values less than 0.25 .

\section{Explanatory variables of the likelihood} of the one-complex model

Explanatory variables (denoted by $z$ in equation 5 below) of the relative likelihood of using a single complex model include those implied by the specific hypotheses being tested: age, sex, race and ethnicity, years of education, income and Medicaid status, number of known illnesses, perceived general health status and physical functioning, memory loss, difficulty in making decisions, difficulty concentrating, marital status and number of living children. See Appendix for MCBS descriptions.

The physical functioning variable is a summary scale of ADL and IADL items developed by Finch et al. ${ }^{28}$ based on ratio magnitude estimation. 29

\section{Analysis}

Hypothesis tests are based on the relative likelihoods of two binary choice models of seeking care. The decision to seek care is represented by the model

$$
y=\left\{\begin{array}{ll}
0 & \text { if } \max (\lambda) \leq \tau \\
1 & \text { if } \max (\lambda)>\tau
\end{array},\right.
$$

interpreted as The decision is to seek care $(y=1)$ if the evaluation of at least one conceptual complex (i.e. an element of the vector $\lambda$ of complexes) exceeds a determining threshold $(\tau)$. For example, in Panel 1 through Panel 4 of Figure 1, the vector I would comprise 1, 2, 3, and 2 elements, respectively, for the assessment of the indicated complexes. Two choice models are considered: Model 1, a single-complex model for which $\lambda=\lambda$ (a scalar), and Model 2, a two-complex model for which $\lambda=\left(\lambda_{0}, \lambda_{l}\right)^{\mathrm{T}}$. For each observation $i$, the latent vector of evaluations $\lambda$ is modeled as a linear function of variables $x$ and a multivariate normal error vector $\varepsilon$ 
$\lambda_{i}=\mathrm{Bx}_{i}+\varepsilon_{i}$

(2)

Hence, the probability of $y=1$, for a general $K$ element vector of complexes, is

$$
P\left(y_{i}=1 \mid \mathbf{x}_{\mathrm{i}}\right)=1-P\left[\left(\begin{array}{c}
\varepsilon_{i 1} \\
\vdots \\
\varepsilon_{i K}
\end{array}\right) \leq\left(\begin{array}{c}
\tau-\boldsymbol{\beta}_{1}^{\prime} \mathbf{x}_{i} \\
\vdots \\
\tau-\boldsymbol{\beta}_{K}^{\prime} \mathbf{x}_{i}
\end{array}\right)\right]
$$

Estimation of models 1 and 2 is achieved using weighted maximum likelihood, subject to the constraint $B \times 1_{J}=1_{J}$ to specify variance (similar to the identifying constraint used by Chiou and Müller). ${ }^{30}$ Although, models with $K>2$ are plausible, they were computationally too expensive for the present work; hence, we restrict analysis to the 1-component and 2 component models. The vector $\mathrm{x}$ comprises the predictor variables listed in the preceding section. The MCBS cross-sectional sample weights are used. The hypothesis that multiple complexes can underlie the decision behavior among the elderly is tested using a likelihood ratio test. To avoid a significant but trivial result, the null hypothesis is arbitrarily selected as the geometric mean likelihood of the single-complex model is 10 percent worse than the geometric mean likelihood of the multiplecomplex model; hence, the likelihood ratio test is based on a non-central chi-square distribution.

The hypotheses regarding characteristics that differentiate the 1-complex and 2-complex models are investigated based on a model of the relative component likelihoods. The dependent variable in this case is the component relative likelihoods of the two choice models defined for each individual $i$ in the data as

$$
\hat{\xi}_{i 1}=\left(\frac{\hat{\ell}_{i, 1}}{\hat{\ell}_{i, 1}+\hat{\ell}_{i, 2}}\right)
$$

The estimated component likelihoods of observation $i$ are denoted as $\hat{\ell}_{i, 1}$ and $\hat{\ell}_{i, 2}$ for models 1 and 2 respectively. The relative likelihood is modeled as a function of the hypothesized explanatory variables, listed in the preceding section and denoted here as $\mathrm{z}$,

$\hat{\zeta}_{\mathrm{M}, \mathrm{i}}=\frac{1}{1+\mathrm{e}^{-\alpha^{\prime} z_{i}}}+\mathrm{u}_{\mathrm{i}}$

where $u_{i}$ is an error term representing the precision of the estimate with expected value equal to the bias, which is asymptotically zero. The error $u_{i}$ is a linear function of a beta-distributed variable with a single parameter $\theta$ : i.e., $u_{i}=2 \times v_{i}-1$ and $v_{i} \sim \operatorname{Beta}(\theta, \theta)$. The parameters $\alpha$ and $\theta$ are estimated via maximum likelihood based on the beta distribution of $v$.

\section{Results}

\section{Sample characteristics}

The sample comprised 2,020 respondents with an average age of 75 years, 57 percent women, 90 percent white, 5 percent Hispanic, and 97 percent having been married at least once. The sample respondents had an average of a high school education and median annual income of $\$ 15,000$ to $\$ 20,000$. Sixty nine percent sought care on their own initiative in 1997 (Table 1).

\section{General hypothesis: one complex model versus two complex model}

The 2-complex model provides a statistically significant, nontrivial (i.e. at least a $10 \%$ improvement in the geometric mean of the likelihood) improvement over the 1-complex model $\mathrm{P}<0.001$ ) in explaining decision behavior among the elderly.

\section{Characteristic-specific hypotheses}

Table 2 shows results providing evidence regarding hypotheses for individual characteristics.

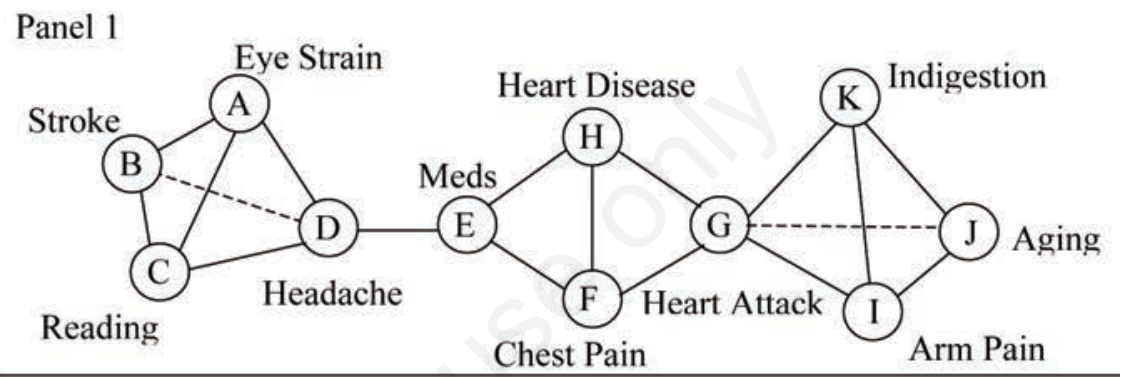

Panel 2

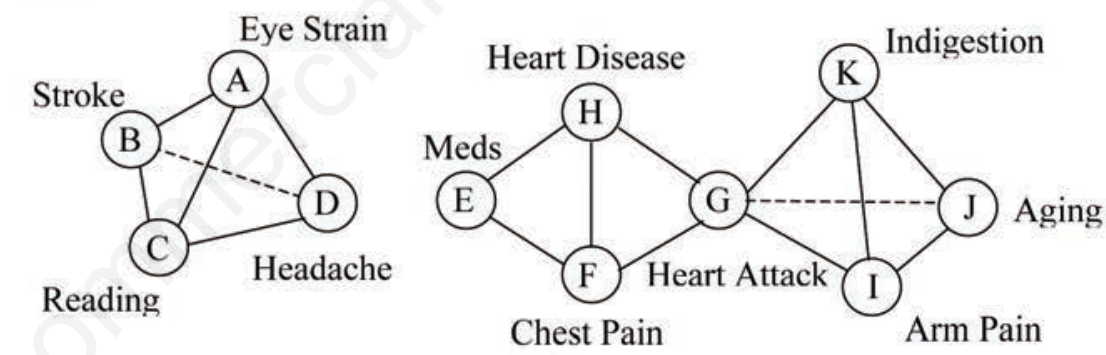

Panel 3

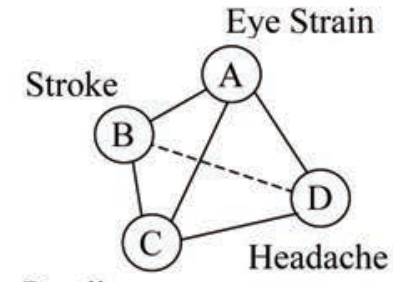

Reading

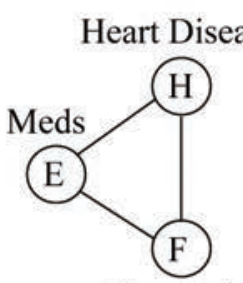

Chest Pain

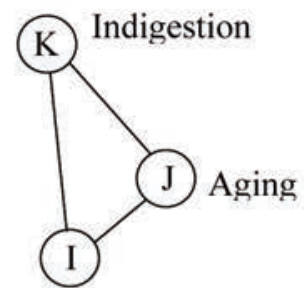

Arm Pain

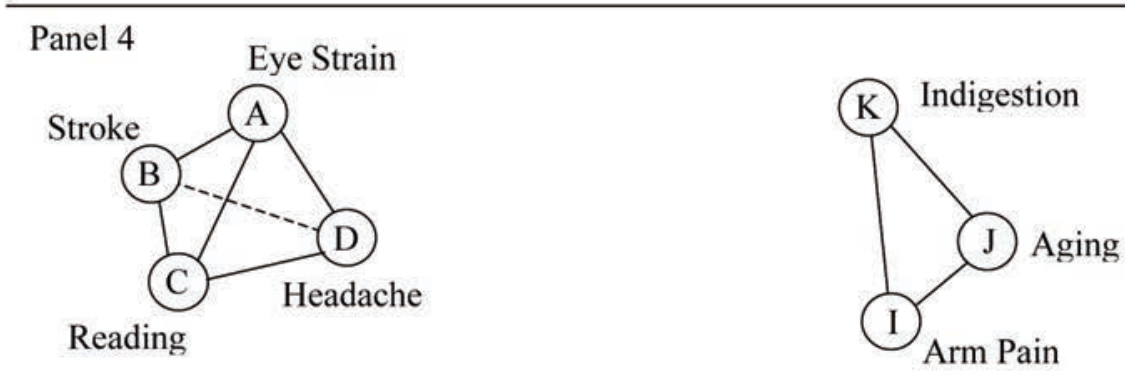

Figure 1. Differentiated conceptual complexes. Each panel depicts a set of conceptual complexes. Panels 2,3, and 4 are derived from Panel 1 by deletion of concepts. 


\section{Age}

The hypothesis that age corresponds to a non-zero effect differentiating the relative likelihood of the one to two complex models is supported $(\mathrm{P}=0.022)$. The direction of effect is such that older persons have a higher likelihood for the 1-complex model; that is, the elderly are more likely to integrate information.

\section{Sex}

The presented theory suggests women generate more elementary and relational concepts than men, implying a greater likelihood of generating a single conceptual complex. Results do not support this hypothesis in terms of a direct effect $(\mathrm{P}=0.539)$. However, sex interacts with characteristics as discussed below, which conforms to the more general proposition that cognitive processing is differentiated by sex.

\section{Culture}

The hypothesis that there is an effect due to culture is supported $(\mathrm{P}=0.002$ for race and 0.001 for ethnicity). In order of increasing tendency to integrate information are Black,
Other, Asian and Pacific Islanders, White, and American Indian. Being of Hispanic ancestry implies a greater relative likelihood of the 1complex model.

\section{Education}

The hypothesis that education differentiates the models is supported $(\mathrm{P}<0.001)$. The negative direction of effect indicates the 1-complex model better explains those with lower education levels and the 2-complex model better explains persons with higher education.

\section{Financial status}

The hypothesis that financial status differentiates the models is supported $(\mathrm{P}=0.001)$. Results also conformed to the expectation that the direction of effect for financial status is the same as that for education. Medicaid status is not significant; however, this is not too surprising as once education and income level are accounted for, Medicaid status is not likely to provide much additional explanatory power.

\section{Number of illnesses}

The hypothesis that those with more known illnesses are more likely to integrate information is supported $(\mathrm{P}<0.001)$. The positive direction of effect implies that the 1-complex model more likely fits persons with greater number of illnesses.

\section{General health and physical functioning}

The hypothesis that perceived health status and level of physical functioning encourage the structuring of health related mental representations is supported $(\mathrm{P}=0.012$ for men and 0.028 for women). However, the directions of effect are opposite: better perceived health corresponds to a lower likelihood of the 1-complex model for men and a higher likelihood of the 1complex model for women. Physical functioning is significant for women $(\mathrm{P}=0.041)$ but not men $(\mathrm{P}=0.543)$. Mostly, in conformance with the result of perceived health, decreased physical functioning is associated with decreased likelihood of the 1-complex model for women (five of the six effect coefficients are negative).

Table 1. Descriptive statistics of key variables. Mean and standard deviations are reported for continuous, count, and dichotomous variables; the median category and the $25^{\text {th }}$ and $75^{\text {th }}$ percentile categories are reported for ordered categorical variables.

\begin{tabular}{|c|c|c|c|c|}
\hline & Mean/median category & $\begin{array}{c}\text { Standard deviation } \\
\text { (25 } 5^{\text {th }} \text { percentile; } 75^{\text {th }} \text { percentile) }\end{array}$ & Minimum & Maximum \\
\hline Age & 75 & 7 & 65 & 98 \\
\hline Females & 0.57 & 0.5 & 0 & 1 \\
\hline $\begin{array}{l}\text { Race } \\
\text { White } \\
\text { American Indian } \\
\text { Asian/Pacific Is. } \\
\text { Black } \\
\text { Other } \\
\text { Hispanic }\end{array}$ & $\begin{array}{l}0.90 \\
0.01 \\
0.01 \\
0.08 \\
0.01 \\
0.05\end{array}$ & $\begin{array}{l}0.30 \\
0.07 \\
0.11 \\
0.26 \\
0.09 \\
0.22\end{array}$ & $\begin{array}{l}0 \\
0 \\
0 \\
0 \\
0 \\
0\end{array}$ & $\begin{array}{l}1 \\
1 \\
1 \\
1 \\
1 \\
1\end{array}$ \\
\hline Years of education & 12 & 4 & 1 & 18 \\
\hline Income & $(15,000-20,000 \$)$ & $(10,000-15,000 \$ ; 30,000-35,000 \$)$ & Less than $\$ 5000$ & Greater than $\$ 50,000$ \\
\hline Medicaid & 0.07 & 0.25 & 0 & 1 \\
\hline $\begin{array}{l}\text { Marital Status } \\
\text { Married } \\
\text { Widowed } \\
\text { Divorced } \\
\text { Separated } \\
\text { Never married }\end{array}$ & $\begin{array}{l}0.55 \\
0.35 \\
0.06 \\
0.01 \\
0.04\end{array}$ & $\begin{array}{l}0.5 \\
0.48 \\
0.24 \\
0.08 \\
0.19\end{array}$ & $\begin{array}{l}0 \\
0 \\
0 \\
0\end{array}$ & $\begin{array}{l}1 \\
1 \\
1 \\
1\end{array}$ \\
\hline No. of children & 2.93 & 2.15 & 0 & 18 \\
\hline $\begin{array}{l}\text { Primary care visit } \\
\text { General health } \\
\text { No. of illnesses } \\
\text { Physical functioning } \\
\text { Difficulty stooping } \\
\text { Difficulty lifting } \\
\text { Difficulty reaching } \\
\text { Difficulty writing } \\
\text { Difficulty walking } \\
\text { Functional ADL scale }\end{array}$ & $\begin{array}{c}0.69 \\
\text { Very Good } \\
1.81 \\
\\
\text { A little } \\
\text { No } \\
\text { No } \\
\text { No } \\
\text { No } \\
-0.27\end{array}$ & $\begin{array}{c}0.46 \\
\text { (Good, Excellent) } \\
1.55 \\
\\
\text { (No; some) } \\
\text { (No; a little) } \\
\text { (No; a little) } \\
\text { (No; a little) } \\
\text { (No; some) } \\
0.55\end{array}$ & $\begin{array}{c}0 \\
\text { Poor } \\
0 \\
\\
\text { No } \\
\text { No } \\
\text { No } \\
\text { No } \\
\text { No } \\
-0.45\end{array}$ & $\begin{array}{c}1 \\
\text { Excellent } \\
10 \\
\text { Not able to do } \\
\text { Not able to do } \\
\text { Not able to do } \\
\text { Not able to do } \\
\text { Not able to do } \\
5.7\end{array}$ \\
\hline $\begin{array}{l}\text { Cognitive functioning } \\
\text { Memory loss } \\
\text { Trouble making decisions } \\
\text { Trouble concentrating }\end{array}$ & $\begin{array}{l}0.94 \\
0.98 \\
0.94\end{array}$ & $\begin{array}{l}0.24 \\
0.15 \\
0.24\end{array}$ & $\begin{array}{l}0 \\
0 \\
0\end{array}$ & $\begin{array}{l}1 \\
1 \\
1\end{array}$ \\
\hline
\end{tabular}




\section{Cognitive functioning}

The hypothesis that cognitive functioning is associated with the structure of conceptual complexes is supported for men but not women $(\mathrm{P}=0.005$ and 0.609 for men and women respectively).

\section{Social support}

The hypothesis that the presence or absence of a spouse and number of children differentiates the 1-complex model from the 2-complex model is supported for spouse $(\mathrm{P}<0.001)$ but not number of children $(\mathrm{P}=0.915)$. The direction of the spouse effect generally indicates 1complex model is favored more by those with a spouse (all coefficients are negative for the marital indicators).

\section{Discussion}

Results suggest some general conclusions: first, the proposition that decision-making can be based on the assessment of multiple cognitive objects is supported.

Second, older persons are more likely to integrate information; more precisely, older persons are more likely to seek care based upon considering a single integrated conceptual complex. This suggests that the inability to filter irrelevant information outweighs the inability to recall and maintain concepts in seeking care. Third, results indicate individual characteristics that differentiate single and multiple complex models. For older persons' decisions to seek care at a doctor's office, the characteristics associated with an increased likelihood of integrating information are older age, lower education, worse health for men and better general health for women, greater memory loss for men, less trouble concentrating and less trouble making decisions for men, more known illnesses, racial category, being of Hispanic ancestry, lower income, and being married.

Contrary to the hypothesized effect, sex did not directly differentiate the models. However, the sex interactions suggest a potential explanation. Perceived health status was significant for both men and women but in different directions: men who perceived themselves as healthy were more likely to segregate information whereas women who perceived themselves as healthy were more likely to integrate information. To the extent that self-reported health status reflects actual status, this result supports the posited hypothesis among healthy adults but not for those in poor health. Similarly, although physical functioning was not significant in men, it was significant in

Table 2. Characteristics influencing the relative likelihood of the one complex to two complex models (n=2020). Results are reported for men and women; the entry Same in the women's columns indicates there is no significant interaction with sex and the effect is the same as reported in the corresponding men column.

\begin{tabular}{|c|c|c|c|c|}
\hline \multirow[t]{3}{*}{ Constructs } & \multicolumn{3}{|c|}{ Coefficients } & \\
\hline & \multicolumn{2}{|c|}{ Men } & & \\
\hline & Effect & $\mathbf{P}$ & Effect & $\mathbf{P}$ \\
\hline Sex & 0.0230 & 0.539 & Same & Same \\
\hline Age & 0.0008 & $0.022 *$ & Same & Same \\
\hline Education & -0.0060 & $<0.001^{*}$ & Same & Same \\
\hline General health & -0.0079 & $0.012^{*}$ & 0.0068 & $0.0288^{*}$ \\
\hline \multicolumn{5}{|l|}{ Physical functioning } \\
\hline Difficulty stooping & -0.0019 & 0.542 & -0.0019 & $0.0413^{*}$ \\
\hline Difficulty lifting & 0.0035 & & -0.0004 & \\
\hline Difficulty reaching & -0.0010 & & -0.0035 & \\
\hline Difficulty writing & -0.0027 & & 0.0019 & \\
\hline Difficulty walking & -0.0002 & & -0.0058 & \\
\hline ADL Scale & 0.0158 & & -0.0039 & \\
\hline \multicolumn{5}{|l|}{ Cognitive functioning } \\
\hline Memory loss & 0.0835 & $0.005^{*}$ & 0.0116 & 0.6088 \\
\hline Problems decisions & 0.0119 & & -0.0251 & \\
\hline Trouble concentrating & -0.0347 & & 0.0146 & \\
\hline Number of illnesses & 0.0064 & $<0.001^{*}$ & Same & Same \\
\hline \multicolumn{5}{|l|}{ Culture, race } \\
\hline American Indian & 0.0204 & $0.002 *$ & Same & Same \\
\hline Asian/Pacific Is. & -0.0050 & & & \\
\hline Black & -0.0644 & & & \\
\hline Other & -0.0279 & & & \\
\hline Hispanic ancestry & 0.0611 & $0.001^{*}$ & Same & Same \\
\hline \multicolumn{5}{|l|}{ Financial status } \\
\hline Income & -0.0028 & $0.001^{*}$ & Same & Same \\
\hline Medicaid status & 0.0098 & 0.258 & Same & Same \\
\hline \multicolumn{5}{|l|}{ Social support, marital status } \\
\hline Single & -0.0352 & $<0.001^{*}$ & Same & Same \\
\hline Widowed & -0.0329 & Same & Same & \\
\hline Divorced & -0.0298 & Same & Same & \\
\hline Separated & -0.0610 & Same & Same & \\
\hline Number of children & 0.0001 & 0.915 & Same & Same \\
\hline Constant & -0.1020 & $0.001^{*}$ & Same & Same \\
\hline
\end{tabular}

*P-values are based on t-statistics for single coefficients and F-statistics for multiple coefficients. 
women, and again, the direction of effect suggested that women in good physical functioning are more likely to integrate information. Generally, the advantage of women over men in working memory access of more contextual concepts in information processing, 15 may disappear or even reverse with ill health: a question that has yet to be studied. For men, reported memory loss and problems making decisions indicated a greater likelihood of integrating information, whereas trouble concentrating indicated a greater likelihood of segregating information. In the present context, this switch in direction is hard to interpret. It may suggest that concentration is necessary to build conceptual structures (to relate concepts), but for men the decision making process is one of analysis by which conceptual pruning leads to segregated conceptual complexes.

The study presented here investigated rudimentary structuring of conceptual complexes and was unable to represent detailed information regarding variation in the structure and content of conceptual complexes. The window of time that captures deliberation and decision is treated as a unit and does not represent the dynamic nature of cognition and decisionmaking.

The observational design and the cross-sectional nature of the data imply that the statistical component of the study describes population-level distributional characteristics. Study conclusions should be recognized as awaiting an experimental design and longitudinal data to provide clearer evidence. A weak variable is the use of number of children in the social support construct. Although it is plausible that having more children increases the probability of greater social support from children, it is also likely that considerable variation exists and the correlation between number of children and social support is small.

Future research is needed that represents individual, decision specific, conceptual complexes. How to represent such structure remains a question. Future investigation into the connection between utilization and inappropriate structuring of conceptual complexes is required to identify the need for interventions that address the structure of conceptual complexes. Also, research into psychological mechanisms that influence organization of conceptual complexes is needed to identify potential points of intervention. Both underutilization and overutilization may be a consequence of the inappropriate structuring of information such as the inappropriate assessment of fewer complexes rather than a more appropriate assessment of more complexes or the inappropriate assessment of many complexes. Future research is needed to identify predictable structure in the individual-contingent nature of this problem.

\section{Conclusions}

Results of this study support the conclusion that older persons are more likely to integrate information, and individuals with identifiable characteristics are more likely to do so than others. The theory tested in this study implies a potential explanation for misutilization of care (either over or under-utilization). Specifically, the inappropriate assessment of single complexes rather than more appropriate multiple complexes, or the inappropriate assessment of multiple complexes rather than more appropriate single complexes can leading individuals to attend to inaccurate or insufficient information thereby possibly generating mistaken judgments.

\section{References}

1. Kaplan MF. Information integration in social judgment: interaction of judge and informational components. In: Kaplan MF, Schwartz S, eds. Human judgment and decision processes. New York: Academic Press; 1975. pp 139-171.

2. Shanteau J. An information-integration analysis of risky decision making. In: Kaplan MF, Schwartz S, eds. Human judgment and decision processes. New York: Academic Press; 1975. pp 109-37.

3. Gigerenzer G, Selten R, eds. Bounded rationality: the adaptive toolbox. Cambridge: MIT Press; 2002.

4. Gigerenzer G, Todd PM, Group AR. Simple heuristics that make us smart. Stich S, ed. New York: Oxford University Press; 1999.

5. Rothrock L, Kirlik A. Inferring rule-based strategies in dynamic judgment tasks: Toward a noncompensatory formulation of the Lens Model. IEEE Transactions on Systems, Man and Cybernetics-Part 1: Systems and Humans 2003;33:58-72.

6. Elrod T, Johnson RD, White J. A new integrated model of noncompensatory and compensatory decision strategies. Organ Behav Hum Decis Process 2004;95:1-19.

7. Medin DL, Lynch EB, Solomon KO. Are there kinds of concepts? Annu Rev Psychol 2000;51:121-47.

8. McGinn C. Conceptual causation: some elementary reflections. Mind 1991;4:573-86.

9. Grady CL. Functional brain imaging and agerelated changes in cognition. Biol Psychol 2000;54:259-81.

10. Viskontas IV, Morrison RG, Holyoak KJ, et al. Relational integration, inhibition, and analogical reasoning in older adults. Psychol Aging 2004;19:581-91.

11. Schacter DL, Norman KA, Koutstaal W. The cognitive neuroscience of constructive mem- ory. Annu Rev Psychol 1998;49:289-318.

12. Naveh-Benjamin M. Adult age differences in memory performance: tests of an associative deficit hypothesis. J Exp Psychol 2000;26:1170-87.

13. Johnson MK, Reeder JA, Raye CL, Mitchell KJ. Second thoughts versus second looks: an age-related deficit in reflectively refreshing just-activated information. Psychol Sci 2002;13:64-7.

14. Geary DC, Saults SJ, Liu F, Hoard MK. Sex differences in spatial cognition, computational fluency, and arithmetical reasoning. J Exp Child Psychol 2000;77:337-53.

15. Larsson M, Lövdén M, Lars-Göran N. Sex differences in recollective experience for olfactory and verbal information. Acta Psychol (Amst) 2003;112:89-103.

16. Weber EU, Hsee CK. Culture and individual judgement and decision making. Appl Psychol 2000;49:32-61.

17. Weber EU, Hsee C. Cross-cultural differences in risk perception, but cross-cultural similarities in attitudes towards perceived risk. Manag Sci 1998;44:1205-17.

18. Yates JF, Lee JW, Bush JG. General knowledge overconfidence: cross-national variations, response styles, and Reality. Organ Behav Hum Decis Process 1997;70:87-94.

19. Choi I, Nisbett RE, Smith EE. Culture, category salience, and inductive reasoning. Cognition 1997;65:15-32.

20. Nisbett RE, Peng K, Choi I, Norenzayan A. Culture and systems of thought: holistic versus analytic cognition. Psychol Rev 2001;108:291-310.

21. DiMaggio P. Culture and cognition. Annu Rev Sociol 1997;23:263-87.

22. VanLehn K. Cognitive skill acquisition. Annu Rev Psychol 1996;47:513-39.

23. Eviatar Z. Culture and brain organization. Brain $\operatorname{Cog}$ 2000;42:50-2.

24. Koriat A, Goldsmith M, Pansky A. Toward a psychology of memory accuracy. Annu Rev Psychol 2000;51:481-537.

25. Iwashyna TJ, Christakis NA. Marriage, widowhood, and health-care use. Soc Sci Med 2003;57:2137-47.

26. Langa KM, Chernew ME, Habeto MU, Katz SJ. The explosion in paid home health care in the 1990's: who received the additional services? Med Care 2001;39:147-57.

27. Mitchell J, Krout JA. Discretion and service use among older adults: the behavioral model revisited. The Gerontologist 1998;38:159-68.

28. Finch M, Kane RL, Philp I. Developing a new metric for ADLs. J Am Geriatr Soc 1995;43:877-84.

29. Narens L. A theory of ratio magnitude estimation. J Math Psychol 1996;40:109-29.

30. Chiou JM, Müller HG. Estimated estimating equations: semiparametric inference for clustered and longitudinal data. J Royal Stat Soc B 2005;67:531-53. 